\title{
Análise de risco como medida preventiva de inundações na Amazônia: estudo de caso de enchente de 2000 em Laranjal do Jari-AP, Brasil
}

\author{
Risk analysis as a preventive measure of floods in the Amazon: a case study of flood \\ in 2000 the Laranjal do Jari-AP, Brazil
}

\author{
Alzira Marques Oliveira', Alan Cavalcanti da Cunha ${ }^{2}$ \\ 1,2 Departamento de Meio Ambiente e Desenvolvimento, UNIFAP
}

\begin{abstract}
Resumo
O objetivo da pesquisa foi testar um modelo conceitual simplificado de contingência para análise de risco de desastre causado pela enchente histórica no município de Laranjal do Jari-AP no ano 2000. A base metodológica foi elaborada em termos de probabilidade e nível dos impactos do evento, considerando a área urbana afetada, e prejuízos econômico-financeiros associados ao evento, incluindo número de desabrigados e vítimas fatais. Estas variáveis foram utilizadas na composição de indicadores escalonados com pesos entre 1 e 5 na referida tabela de contingência. O resultado da soma dos escores medidos de cada fator de impacto (multiplicação do peso pela nota de avaliação) serviram como referência de cálculo da probabilidade de recorrência de novos eventos extremos, considerando-se principalmente o grau de exposição na área afetada. Além disso, a matriz de riscos gerada registrou altos níveis de probabilidade de ocorrência e gravidade do impacto no evento. Considerando os níveis de criticidade socioambiental do evento extremo, foi quantificado o nível de vulnerabilidade da comunidade com indicadores elevados em relação à fragilidade da comunidade. Concluímos que os indicadores da matriz podem ser utilizados como referência futura às medidas adotadas no médio e curto prazos embasadas em sistemas de monitoramento e gestão.
\end{abstract}

Palavras-chave: Análise de risco. Subsídio à decisão. Mitigação. Adaptação. Crise

\begin{abstract}
The objective of the investigation was to test a simplified conceptual model of contingency for risk analysis of flood disaster in the city of Laranjal do Jari - AP during the year 2000. The methodological basis was elaborated in terms of probability and level of impact of the event, considering the urban area affected, as well as economic and financial losses associated with it, including homeless and number of fatal victims. These variables were used in the composition of weights indicators ranging between 1 to 5 obtained from the contingency table. The sum of measured scores of each impact factor (multiplying the weight versus the evaluation score) served as a support for calculating the probability of recurrence of extreme event, principally considering the degree of exposure of the area in relation to the margins of the Jari river. Furthermore, the risk matrix generated charged columns and rows that scored highest levels of likelihood and severity of impact event. Whereas the levels of environmental criticality of extreme event. Then, it was quantified the level of vulnerability of the community and the fragility indicators of the affected community. We conclude that the indicators of the matrix can be used as subsidies to preventives measures adopted in future in the medium and short term, based on crisis monitoring and management.
\end{abstract}

Keywords: Risk analysis. Decision support. Mitigation. Adaptation. Crisis 


\section{Introdução}

Na literatura internacional risco é a probabilidade de ocorrer consequências danosas ou perdas esperadas, como resultado de interações entre um perigo natural e as condições de vulnerabilidade local (UNDP, 2004)

Para a Defesa Civil brasileira risco é a probabilidade de ocorrência de um acidente ou evento adverso, relacionado com a intensidade dos danos ou perdas, resultantes dos mesmos (BRASIL, 2007). Segundo Veyret (2007) "risco" se define como uma situação relacionada à percepção de uma possível catástrofe, na qual uma população ou um indivíduo a percebe e pode sofrer seus efeitos. Zanirato et al. (2008) descrevem que o "risco não é algo apenas a ser medido, mas pode ser apreendido e qualificado na perspectiva da sociedade do medo e fundamentado na tipologia do risco. Trata-se, portanto, de um evento cultural que remete para além da condição de indivíduo".

Em linhas gerais, a ocorrência do risco pode ser observada mediante a associação de duas variáveis: a vulnerabilidade da população, como um processo socialmente construído (artificial), e a susceptibilidade local à dinâmica planetária (natural) (VEYRET, 2007; CUTTER et al. 2003).

Segundo Tobin e Montz (1997) o risco ainda pode ser visto como resultado de uma probabilidade de ocorrência de eventos particulares e de previsão de perda, devendo ser avaliado de acordo com tendências históricas. Portanto, a informação gerada pela tendência histórica é um componente importante para avaliar o risco técnico. Não necessariamente traduz ou indica o número de exposição de um perigo ou as perdas esperadas por um evento específico.

Salgado (2005) assevera que o risco é a probabilidade de ocorrência multiplicada pela vulnerabilidade. Esta proposição foi utilizada em 1994 para avaliar risco de terremoto na Nova Zelândia. Neste caso, definiram probabilidade como a chance de um terremoto ocorrer (baseado em resultados de um modelo sísmico), e vulnerabilidade como o potencial de danos causados à sociedade. Enquanto esta fórmula simples representa uma tentativa útil de incluir fatores adicionais que afetam o risco, apresenta restrições por não incorporar diferenças geográficas em função do tamanho da população e densidade (ou exposição), além de fatores como adaptação comum em reduzir perdas de vidas humanas (TOBIN e MONTZ, 1997; BLAIKIE, 1994).

Contudo, de modo geral são aceitáveis outras expressões simples com o objetivo de melhor descrever um resultado associado ao risco, por exemplo, a equação apresentada pela Defesa Civil (BRASIL, 2007), cuja definição seria:

$$
\mathrm{R}=\mathrm{A}+\mathrm{V}
$$

sendo:

$\mathrm{R}=$ Risco

$\mathrm{A}=$ Ameaça
$\mathrm{V}=$ Vulnerabilidade

Em combinação, esta relação serve para explicar a interação com a magnitude do evento ou acidente, que defina os efeitos adversos medidos em termos de intensidade dos danos previstos. No entanto, observa-se que há vários fatores que induzem uma determinada população a uma situação de risco. Neste caso, é importante analisar as principais características de um cenário. A este processo metodológico denomina-se de caracterização do risco (VEYRET, 2007). O processo de caracterização do risco objetiva o conhecimento dos fatores de risco que afetam o território, identificando a localização, a gravidade dos danos potenciais e as probabilidades de ocorrência (BRASILIANO, 2011; CASTRO, 2008; VEYRET, 2007).

As cidades com espaços hegemônicos, de concentração urbana, de acúmulo de população e de complexas (e inadequadas) infra-estruturas, tornam-se, espaços onde indivíduos e sociedade encontram-se mais vulneráveis a perdas advindas de processos variados. Isto é, espaços de risco (SANTOS, 1998; ADGER, 2006).

Nestes espaços, o risco também pode ocorrer, frequentemente, em função da inadequação ou de características conflitantes das formas de ocupação e uso do solo e os processos produtivos/tecnológicos, sociais e "naturais". Estes fatores determinam situações de perdas potenciais ou efetivas, pela apropriação e uso dos recursos naturais mediante processos produtivos e em função da própria dinâmica natural do ambiente, os quais tendem a gerar riscos à sociedade, relacionando-se efetivamente à sua dinâmica sócio-espacial (VEYRET, 2007; SANTOS, 1998).

Nesse contexto surge como ferramenta preventiva a análise de risco com pressupostos assentados em diferentes etapas e procedimentos. Nestes pressupostos, as propostas podem ser mencionadas, sendo apenas adaptadas às suas especificidades temáticas ou de origem disciplinar. É importante salientar que a análise de risco tem como fim fornecer informações científicas para a tomada de decisão. Ou seja, a análise do risco é considerada como uma ferramenta de elaboração de política pública frente aos desastres (ZIMMERMAN, 1986; BLAIKIE, 1996; COBURN et al. 1991).

Com base nesses pressupostos o objetivo da presente investigação foi testar um modelo conceitual simples de contingência para analisar o risco de desastre e a probabilidade e o impacto relacionado à enchente histórica ocorrida no município de Laranjal do Jari-AP no ano 2000.

\section{Material e métodos}

\section{1 Área de Estudo}

A área atingida pelas frequentes enchentes é a capital do município homônimo no sul do Estado do Amapá, Laranjal do Jarí. O município foi criado em 17 de dezembro de 1987, tendo suas origens vinculadas a episódios da história de colonização do Rio Jari e às influências socio- 


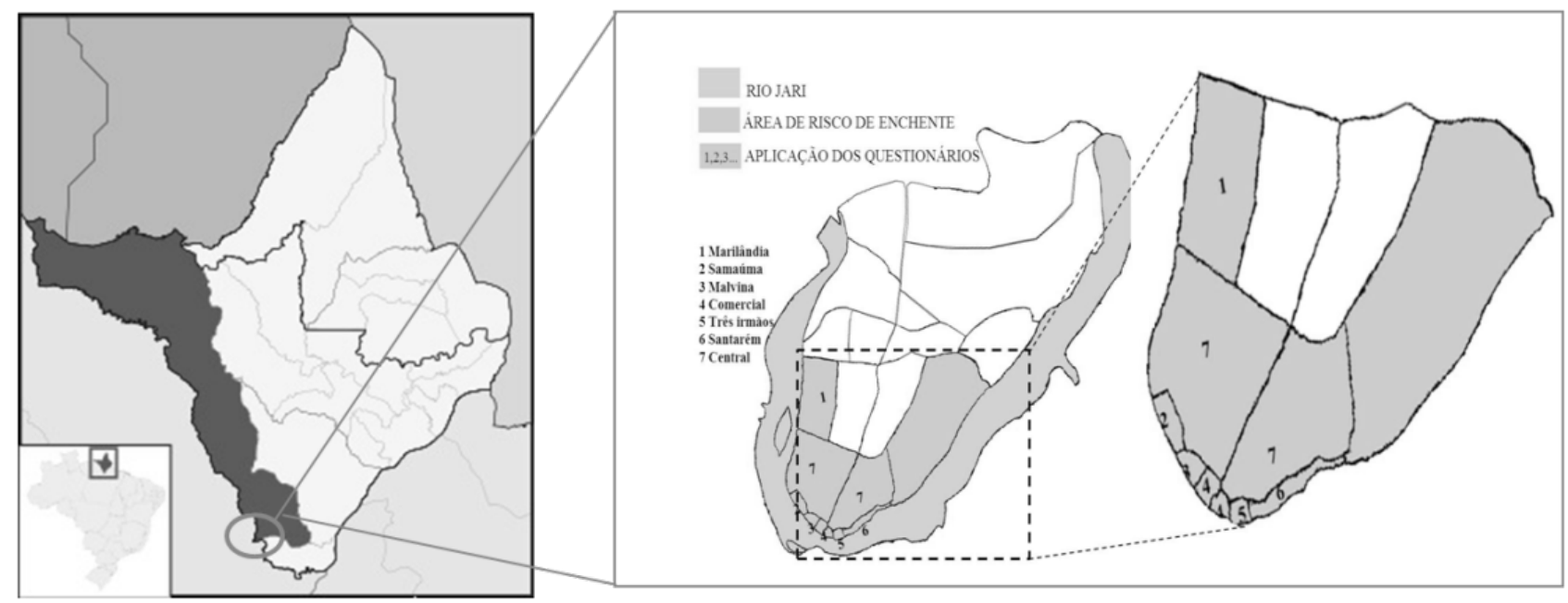

Figura 1 - Localização do município de Laranjal do Jari. À direita, destaque, na cor cinza para os bairros sujeitos às frequentes enchentes

econômicas decorrentes da implantação e funcionamento do Projeto Jari Florestal (OLIVEIRA e CUNHA, 2010).

A zona urbana insere-se em área de várzea e é composta por sete bairros: Mirilândia, Samaúma, Malvina, Comercial, Três irmãos, Santarém e Central (Figura 1)

O destaque em cinza na Figura 1, indica os bairros de várzea urbana de Laranjal do Jari, os quais são os mais afetados pelas enchentes. De acordo com dados do Plano Diretor Participativo de Laranjal do Jari, essa área corresponde a $49 \%$ da população total desse município, o que equivale a 19.571 habitantes (BRITO PAIXÃO e TOSTES, 2010; IBGE, 2010). Historicamente os eventos climáticos adversos mais frequentes nesse município foram as enchentes de 2000, 2006 e 2008, sendo considerados como de grandes proporções (Quadro 1).

Segundo Lucas et al. (2010) e Oliveira e Cunha (2010) hidrologicamente a enchente do ano de 2000 foi consequência das anomalias positivas de chuva ao longo de cinco meses do período chuvoso. Observa-se que a intensidade das chuvas combinada com sua longa duração (ver $3^{a}$ coluna e $1^{\text {a }}$ linha, com 50 dias no Quadro -1) impactou fortemente o regime hidrológico da bacia, causando danos e prejuízos econômicos e sociais extraordinários e, até a atualidade, ainda insuperáveis, ao município de Laranjal do Jari-AP.

Os impactos socioeconômicos da enchente de 2000, sendo os mais graves da história de desastre natural de todo o Amapá, sofreram interferência gerencial do Estado no município e auxílio emergencial do governo federal. Por exemplo, os prejuízos econômicos e sociais somados nos três eventos foram superiores ao Produto Interno Bruto do próprio município (PIB) daquele ano, sendo necessário decretar estado de calamidade pública (OLIVEIRA e CUNHA, 2010).

Quadro1 - Característica do evento e repercussão social

\begin{tabular}{|c|c|c|c|c|c|c|}
\hline \multirow[b]{2}{*}{ Ano } & \multicolumn{2}{|c|}{ Nível do rio (m) } & \multirow[b]{2}{*}{ Duração (dias) } & \multicolumn{2}{|c|}{ Prejuízos (R\$) } & \multirow[b]{2}{*}{ Danos Humanos } \\
\hline & Normal & $\begin{array}{l}\text { Observado } \\
\text { na Enchente }\end{array}$ & & Econômicos & Sociais & \\
\hline 2000 & & 4,00 & 50 & 7,6 milhões & 4,2 milhões & $\begin{array}{l}12.983 \text { desalojados, } 6.384 \text { desabri- } \\
\text { gados, } 3.978 \text { pessoas levemente feri- } \\
\text { das, } 111 \text { gravemente feridos e } 2.407 \\
\text { pessoas enfermas e } 1 \text { morte }\end{array}$ \\
\hline 2006 & & 2,46 & 8 & 905 mil & 675,3 mil & 655 desalojados e 285 desabrigados \\
\hline 2008 & & 3,45 & 30 & 2,0 milhões & $640 \mathrm{mil}$ & $\begin{array}{l}\text { 13.045 desalojados, } \\
2.035 \text { desabrigados e } 1 \text { morte }\end{array}$ \\
\hline
\end{tabular}

Fonte: Adaptado de AVADAN (2000); MARQUES e CUNHA (2008); QUARESMA (2008) 


\subsection{Método de Análise de Contingência e Ma- triz de Risco}

O método utilizado para análise de risco é baseado em cenários de acidentes associados a cada risco identificado. A aplicação da matriz de risco utiliza a estimativa do grau de gravidade dos danos potenciais e a probabilidade de ocorrência do risco (BRASILIANO, 2011; AUGUSTO FILHO, 2001; ALVES, 2005).

A probabilidade é definida como a chance ou frequência observada de ocorrências com consequências negativas à população, tanto em termos ambientais quanto socioeconômicas. Segundo Brasiliano (2011), Castro (2008) e Veyret (2007) a gravidade é definida como as consequências de um evento, expressas na escala e intensidade das consequências negativas à população, em termos de perda de bens e problemas ambientais.

Associado ao grau de risco (probabilidade $x$ gravidade) está inserido o conceito de vulnerabilidade ou fragilidade, o qual pode ser identificado através dos níveis de fatores de risco (Tabela 1).

\subsection{1 - Dados de campo para avaliação da vulnera- bilidade socioambiental}

Para identificar o nível de vulnerabilidade foram aplicados cento e quarenta e nove (149) formulários em sete bairros da zona urbana do município (Figura-1). A amostragem não foi completamente aleatória, devido às circunstâncias de dificuldade de acesso local e período de coleta. Contudo, foi considerada representativa. Deste modo, para caracterizar o nível de vulnerabilidade local foram consideradas as seguintes variáveis socioeconômicas: renda, fonte de renda, escolaridade, abastecimento de água; e variáveis físico-ambientais: localização exata em relação a distância do rio, material utilizado na construção da residência, destino do lixo e esgotamento sanitário (Tabela 2).

\subsubsection{Critério de Exposição (E)}

Assim como os fatores de risco, o critério de exposição depende de uma escala de valoração que mensura

Tabela 1 - Nível da vulnerabilidade socioambiental

\begin{tabular}{|l|c|}
\hline \multicolumn{1}{|c|}{ Escala } & Pontuação \\
\hline Influencia muito & 3 \\
\hline Influencia medianamente & 2 \\
\hline Influencia muito pouco $\mu$ ou quase nada & 1 \\
\hline
\end{tabular}

Fonte: Adaptada de Brasiliano, 2011

Tabela 2 - Caracterização de vulnerabilidades: variáveis socioambientais e físico-ambientais.

\begin{tabular}{|c|c|c|c|c|c|c|c|c|c|}
\hline \multicolumn{8}{|c|}{ FATORES } & \multirow[b]{2}{*}{ Escore } & \multirow[b]{2}{*}{$\begin{array}{c}\text { Nível de } \\
\text { Vulnerabili } \\
\text { dade }\end{array}$} \\
\hline $\begin{array}{c}\text { Renda } \\
\left(\mathrm{X}_{1}\right)\end{array}$ & $\begin{array}{l}\text { Fonte de } \\
\text { renda }\left(\mathrm{X}_{2}\right)\end{array}$ & $\begin{array}{c}\text { Escolaridade } \\
\left(\mathrm{X}_{3}\right)\end{array}$ & $\begin{array}{l}\text { Locali } \\
\text { zação } \\
\left(\mathrm{X}_{4}\right)\end{array}$ & $\begin{array}{c}\text { Material } \\
\left(\mathrm{X}_{5}\right)\end{array}$ & $\begin{array}{l}\text { Abast. de } \\
\text { água }\left(\mathrm{X}_{6}\right)\end{array}$ & $\begin{array}{l}\text { Dest. do } \\
\text { Lixo }\left(X_{7}\right)\end{array}$ & $\begin{array}{l}\text { Esgoto } \\
\left(\mathrm{X}_{8}\right)\end{array}$ & & \\
\hline $\begin{array}{l}\text { Acima } \\
\text { de } 4 \\
\mathrm{SM}^{1}\end{array}$ & $\begin{array}{c}\text { Vinculo } \\
\text { empregatício; } \\
\text { comerciante, } \\
\text { empresário }\end{array}$ & $\begin{array}{l}\text { Ensino Sup. } \\
\text { Incompleto; } \\
\text { Ens. Sup. } \\
\text { Completo }\end{array}$ & Asfalto & Alvenaria & $\begin{array}{l}\text { Rede Geral } \\
\text { com } \\
\text { canalização }\end{array}$ & $\begin{array}{l}\text { Coleta pela } \\
\text { Prefeitura/pa } \\
\text { rticular }\end{array}$ & $\begin{array}{l}\text { Rede } \\
\text { de } \\
\text { esgoto }\end{array}$ & 1 & Baixa \\
\hline $\begin{array}{l}\text { De } 1 \text { a } \\
3 \text { SM }\end{array}$ & Aposentado & $\begin{array}{c}\text { Ensino Fund. } \\
\text { Completo à } \\
\text { Ensino Médio } \\
\text { Completo }\end{array}$ & Terra & Mista & $\begin{array}{l}\text { Rede Geral } \\
\text { sem } \\
\text { canalização }\end{array}$ & Queima & Fossa & 2 & Média \\
\hline $\begin{array}{l}\text { Até } 1 \\
\text { SM }\end{array}$ & $\begin{array}{l}\text { Sem renda ou } \\
\text { por conta } \\
\text { própria }\end{array}$ & $\begin{array}{l}\text { Nenhum; } \\
\text { alfabetizado; } \\
\text { Ens. Fund. } \\
\text { incompleto }\end{array}$ & $\begin{array}{l}\text { Ponte/ } \\
\text { passarel } \\
\text { a }\end{array}$ & Madeira & $\begin{array}{l}\text { Sem acesso } \\
\text { a água } \\
\text { potável }\end{array}$ & Descarte & $\begin{array}{c}\text { Direto } \\
\text { no rio } \\
\text { ou Céu } \\
\text { aberto }\end{array}$ & 3 & Alta \\
\hline
\end{tabular}

\footnotetext{
${ }^{1}$ SM- Salário Mínimo
} 
a frequência de manifestação dos desastres na região. Estes foram avaliados segundo três óticas: condições passadas, presentes e futuras (BRASIL, 2007). Para valorar o critério de exposição existente em Laranjal do Jari a base da escala ocorreu segundo a Tabela 3.

Segundo Brasiliano (2011) não se deve observar somente o passado, pois se não for assim somente o passado estará realizando uma projeção, não levando em consideração variáveis presentes e futuras, podendo gerar erros estratégicos (LUCAS et al. 2010; SANTOS, 2011).

\subsubsection{Nível de probabilidade (NP)}

O Nível de probabilidade é o resultado da multiplicação do valor final do nível de vulnerabilidade (Tabela 2) versus critério de exposição (Tabela 3), conforme equação (2) abaixo:

$$
\mathrm{NP}=\mathrm{V} \times \mathrm{E}
$$

$O$ resultado dessa equação representa o nível de probabilidade (Tabela-4) em termos de porcentagem da ocorrência de evento.

\subsubsection{Grau de impacto (GI)}

Segundo Brasiliano (2011), Castro (2008), Oliveira e Cunha (2010) e Araújo (2011) a mensuração do impacto de um desastre deve considerar a área afetada, prejuízos, número de desabrigados e vitimas fatais. Para tanto, considerou-se os critérios da Tabela 05.

O nível de impacto é o resultado da soma dos resultados de cada fator de impacto (multiplicação do peso versus nota), dividido pela soma dos respectivos pesos (média ponderada), mostrado a seguir: nível de impacto igual a soma dos pesos $(3+2+4+5) / 14$. Assim, o nível de impacto possui a seguinte classificação conforme

Tabela 3 - Critério de exposição e pontuação dos fatores de risco

\begin{tabular}{|l|l|}
\hline Escala & Pontuação \\
\hline Uma vez/ano ou mais no período de 10 anos & 5 \\
\hline Uma ou mais vezes no período de 20 anos & 4 \\
\hline Uma ou mais vezes no período de 21 e 50 anos & 3 \\
\hline Uma ou mais vezes a cada 100 anos & 2 \\
\hline Uma ou mais vezes a cada 500 anos & 1 \\
\hline
\end{tabular}

Fonte: Brasiliano (2011)

Tabela 4 - Nível de probabilidade

\begin{tabular}{|l|l|c|}
\hline \multicolumn{2}{|c|}{ Escala } & \multicolumn{2}{|c|}{ Nível de Probabilidade \% } \\
\hline $1-3$ & Baixa & 4 a 20 \\
\hline $3,01-6$ & Média & 20,01 a 40 \\
\hline $6,1-9$ & Alta & 40,01 a 60 \\
\hline $9,01-12$ & Muito Alta & 60,01 a 80 \\
\hline $12,01-15$ & Extremamente alta & 80,01 a 100 \\
\hline
\end{tabular}

Fonte: Brasiliano (2011)

Tabela 5 - Critério dos impactos escalonados pelos valores entre 1 (insignificante) e 5 (catastrófico)

\begin{tabular}{|c|c|c|c|c|}
\hline \multirow{2}{*}{ Escala } & Áreas afetadas $\mathbf{( m}^{\mathbf{2}} \mathbf{~}$ & Prejuízos (US\$) & Desabrigados & Vitimas Fatais \\
\cline { 2 - 5 } & $\mathbf{( 3 )}$ & $\mathbf{( 2 )}$ & $\mathbf{( 4 )}$ & $\mathbf{( 5 )}$ \\
\hline 1 & $0-369$ & $10-103$ & $1-148$ & $1-20$ \\
\hline 2 & $370-2729$ & $103-105$ & $149-1096$ & $21-148$ \\
\hline 3 & $2730-20171$ & $105-107$ & $1097-8103$ & $149-1096$ \\
\hline 4 & $20172-149047$ & $107-109$ & $8104-59874$ & $1097-8103$ \\
\hline 5 & $>149048$ & $>109$ & $>59875$ & $>8104$ \\
\hline
\end{tabular}

Fonte: Araújo (2011) 
Tabela 6 - Nível de impacto

\begin{tabular}{|c|l|}
\hline Grau de Impacto & Nível de Impacto \\
\hline $4,51-5,00$ & Catastrófico \\
\hline $3,51-4,50$ & Severo \\
\hline $2,51-3,50$ & Moderado \\
\hline $1,51-2,50$ & Leve \\
\hline $1,00-1,50$ & Insignificante \\
\hline
\end{tabular}

Fonte: Araújo (2011)

mostra a Tabela 6.

\subsubsection{Matriz de risco (MR)}

Com o objetivo de visualizar e, ao mesmo tempo, implementar uma forma de tratamento de cada desastre, o resultado da avaliação dos riscos normalmente é apresentada em forma de uma matriz de risco (Brasiliano, 2011; Araújo, 2011; Veyret, 2007), permitindo o fácil acompanhamento visual acerca da dinâmica dos riscos (Figura 2).

A matriz de riscos mostra os pontos de cruzamento entre linhas e colunas (horizontal e vertical) da probabilidade de ocorrência e do impacto. Quanto maior o valor dos eixos horizontal e vertical, mais severo é o impacto. Desta forma, pela visualização da matriz em quatro níveis de criticidade, é possível avaliar o nível de vulnerabilidade da comunidade em Laranjal do Jari. Por exemplo, o nível 5A representa alto impacto e alta probabilidade de ocorrência, e o nível 1D, o inverso. Assim, quanto maior for a probabilidade e o impacto de um desastre, maior será o nível de fragilidade registrado na Matriz. Os desastres plotados em cada nível devem receber um tratamento específico da Defesa Civil. Desta forma é possível priorizar ações de contingência e de prevenção na comunidade estudada de acordo com as condições monitoradas de ameaças naturais eminentes.

Pela divisão da matriz em quatro níveis de criticidade facilita-se a avaliação do nível de vulnerabilidade da comunidade. Os níveis de desastres plotados na matriz terão tratamento específico, conforme a condição de efetividade de reação social ao evento local. Desta forma priorizam-se ações de contingência e de prevenção na comunidade estudada com base na matriz.

\section{Resultados e discussões}

Segundo Marandola Jr e Hogan (2004), após a identificação do grau de risco, são tomadas as decisões sobre as estratégias de mitigação ao evento. Mitigação de risco pode ser definida como "qualquer ação sustentada para reduzi-los ou eliminá-los a longo prazo, considerando pessoas, bens, perigos e os efeitos adversos decorrentes destes últimos" (VEYRET, 2007; CASTRO, 2008).

A adoção de projetos ou de programas integrados destinados a mitigar os riscos e as vulnerabilidades do território é fundamental para a eliminação ou redução da possibilidade de ocorrência, ou dos efeitos que possam eventualmente resultar de acidente grave ou catástrofe. As estratégias para mitigação de risco incluem diversos instrumentos com a implementação de medidas de ordenamento do território. Estas poderão ser consideradas como instrumentos de mitigação do risco mediante regulação das áreas chaves ou previsão de requalificação das mesmas.
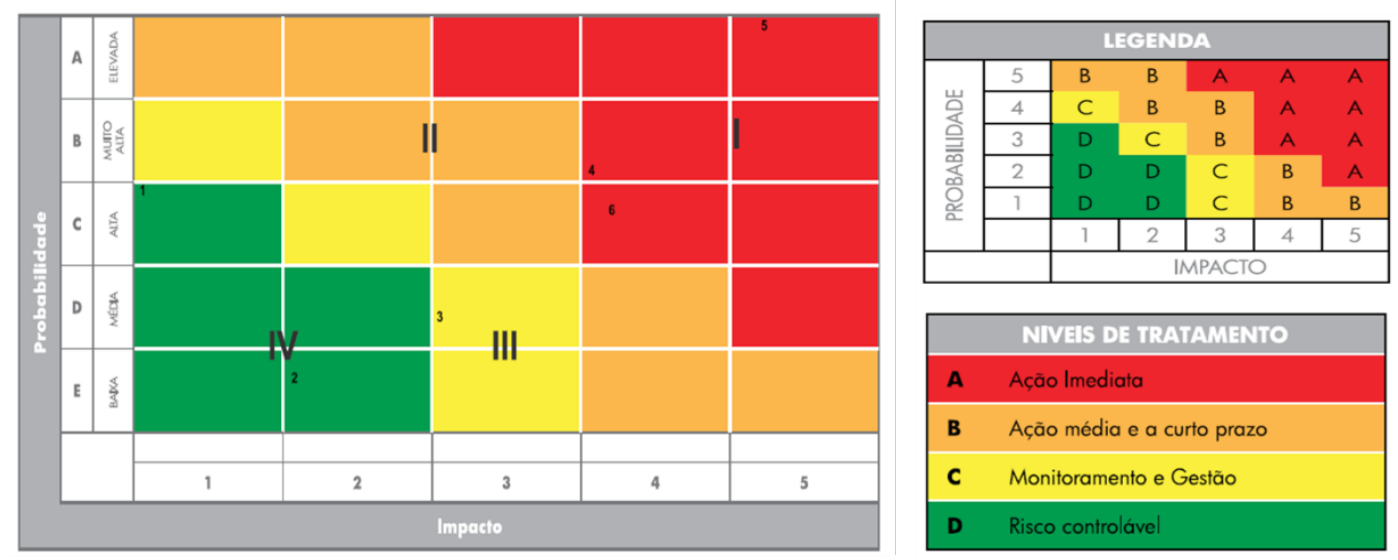

Figura 2 - Matriz de impacto 
Tabela 7: Valores estimados pela análise de risco

\begin{tabular}{|c|c|c|c|c|c|}
\hline & $\begin{array}{c}\text { Nível de } \\
\text { Vulnerabilidade }\end{array}$ & $\begin{array}{c}\text { Critério de } \\
\text { exposição }\end{array}$ & $\begin{array}{c}\text { Grau de } \\
\text { Probabilidade }\end{array}$ & $\begin{array}{c}\text { Critérios } \\
\text { dos impactos }\end{array}$ & $\begin{array}{c}\text { Nível do } \\
\text { impacto }\end{array}$ \\
\hline Valor encontrado & Tabela 2 & Tabela 04 & Tabela 5 & Tabela 6 & Tabela 7 \\
\hline Escala & 3 & 5 & $10,1-15$ & 3,28 & $2,51-3,50$ \\
Influência muito & $\begin{array}{c}3 \text { enchentes nos } \\
\text { últimos } 10 \text { anos }\end{array}$ & $\begin{array}{c}\text { Extremamente Alto } \\
\text { de } 80,01 \text { a } 100 \%\end{array}$ & Moderado \\
\hline
\end{tabular}

A partir da utilização do modelo de análise de risco adaptado de Brasiliano (2011) foi possível determinar a probabilidade e o impacto da ocorrência de desastre relacionada a enchente em Laranjal do Jari-AP em 2000, considerando-se quais medidas deveriam ser adotadas a partir da geração da matriz de risco, elaborada para este fim (Figura-2). Os detalhes da repercussões sociais e econômicas do evento extremo de cheia, segundo Marques e Cunha (2008) e AVADAN (2000), são mostrados na Tabela 1, e os resultados da análise de risco, de acordo com Brasiliano (2001) para o grau de impacto e nível de probabilidade, são apresentados na Tabela 7.

De acordo com a metodologia os valores observados na Tabela 7 foram distribuídos na matriz de risco (Figura 2). Na matriz de risco o desastre de enchente se enquadrou na coluna 3, que correspondeu ao grau de impacto e linha 3 (C) com probabilidade alta. De acordo com a matriz de risco as principais medidas a serem adotadas em uma enchente na bacia hidrográfica devem ser a médio e curto prazos, mediante ações de monitoramento e gestão. Este efeito está identificado na linha 3 e coluna 2 (Figura 2), que corresponde aos níveis de tratamento.

Reforçando esta hipótese, Barth (1997) comenta que as medidas de manejo em bacia urbana devem conciliar funções que os dispositivos naturais têm no ciclo hidrológico com necessidade de expansão urbana, contribuindo com a manutenção da capacidade de retenção das depressões, preservando a permeabilidade do solo e a cobertura vegetal, além de manter suas características de infiltração e retenção, especialmente o armazenamento das várzeas.

O planejamento, desenvolvimento e gestão de um eficiente sistema do drenagem urbana em estratégia de mitigação de inundações ocorrem através de uma bem coordenada e balanceada combinação de medidas não estruturais e estruturais (MAKSIMOVIC e TODOROVIC, 1998; TUCCI e VILLANUEVA, 2001; KUNDZEWICZ, 2002; GRANZIERA, 2001).

Segundo Alves (2005) em geral as medidas não estruturais, por serem preventivas, tendem a ser as mais adequadas para as áreas não desenvolvidas e as estruturais às áreas já urbanizadas. Quando o desenvolvimento da urbanização é considerado, significativos resultados podem ser obtidos com medidas não estruturais de controle de enchentes (TUCCI, 2001).

Segundo Brasiliano (2011) os sistemas de respostas de emergência como um todo ainda não estão adaptados a situação de massa. Isto depende de diversos fatores, como problemas de comando e falta de coordenação e organização do local do evento. Portanto, os fundamentos para enfrentar os desastres estão contidos no processo de gestão de riscos (BRASILIANO, 2011; VEYRET, 2007).

\section{Conclusão}

A presente pesquisa teve como objetivo aplicar um modelo conceitual de contingência para análise de risco de desastre ocorrido em uma área urbana afetada por evento hidrológico adverso na Amazônia Oriental, em Laranjal do Jari-AP. Deste modo, foram quantificadas probabilidades e níveis dos impactos relacionados com a enchente de 2000, com a maior repercussão histórica já registrada no referido município.

Neste caso, adaptou-se o modelo de análise de risco de Brasiliano (2011), e demonstrada sua adequação para estimar simplificadamente a probabilidade de ocorrência de enchente na bacia hidrográfica do rio Jarí. Além disso, avaliou-se o nível de impacto causado pelo evento com projeção para o futuro, caso venha ocorrer novamente.

Desta forma, confirma-se a hipótese elaborada para a presente investigação:

O grau de probabilidade de ocorrência de enchente na bacia do rio Jarí foi extremamente alto, com nível entre 80,01 a 100\%. Portanto, há chances reais de eventos semelhantes ocorrerem futuramente, a aproximadamente cada 5 a 6 anos;

O nível de impacto foi moderado, atingindo parcialmente a área urbana de Laranjal do Jari. A vantagem é que, de acordo com a matriz de risco, o nível moderado permite a minimização de impactos com adoção de medidas preventivas a curto e médio prazos, o que favorece a ação da governança do setor de desastres (Defesa Civil) dos eventos, utilizando-se menos recursos do que se realizados para ações reativas;

Porém, o monitoramento dos fatores climáticos deve ser integrado à gestão do risco, de modo que se sejam utilizadas como ferramentas indispen-sáveis no combate aos impactos de desastres naturais futuros. 


\section{Agradecimentos}

Os autores agradecem a Coordenação Aperfeiçoamento de Pessoal de Nível Superior (CAPES) pelo auxilio financeiro - bolsa de mestrado. Ao projeto SUDAM-IEPA -UNIFAP, convênio 702813/2008 pelo auxílio financeiro para o desenvolvimento da pesquisa e trabalho de campo.

\section{Referências}

ADGER, W.N. Vulnerability. Global Environmental Change, V.16, p.268-81, 2006.

ALVES, Elisânia Magalhães. Medidas não-estruturais na prevenção de enchentes em bacias urbanas: cenários para a bacia do gregório, São Carlos - SP. Dissertação mestrado. Universidade de São Paulo. Escola de engenharia de São Carlos, 2005. Disponível em: www. cipedya.com/web/FileDownload.aspx?IDFile=160215. Acesso em: 24 ago. 2012.

ARAÚJO, S. M. V. G. As áreas de preservação permanente e a questão urbana. Brasília: Consultoria Legislativa da Câmara dos Deputados. 2002.

AUGUSTO FILHO, O. Carta de Risco de Escorregamentos Quantificada em Ambiente de SIG como Subsídio para Planos de Seguro em Áreas Urbanas: Um Ensaio em Caraguatatuba (SP). Tese de doutorado. Instituto de Geociências e Ciências Exatas/Unesp, Rio Claro, SP, rasil, 2001

AVADAN - Avaliação de danos. Defesa Civil - Governo do estado do Amapá. CEDEC-AP: Classificação do porte dos danos (2000).

BARTH, R. T. (1997). Planos Diretores em Drenagem Urbana: Proposição de Medidas para a sua Implementação. Tese (Doutorado) - Escola Politécnica do Departamento de Engenharia Hidráulica e Sanitária, Universidade de São Paulo, São Paulo.

BLIKIE, P. CANNON, T; WISNER, B. At Risk: Natural Hazards, Peoples Vulnerability and Disasters. Routledge, 1994.

BRASIL (2007). Conferência Geral Sobre Desastres: para prefeitos, dirigente de instituições públicas e privadas e líderes comunitários. Ministério da Integração Nacional. Brasília, 2007.

BRASILIANO, Antônio Celso Ribeiro. Um modelo de Análise de Risco para desastre - RJ. Sicurezza. São Paulo, Jan. 2011. Seção Ponto de vista. Disponível em: http://www.brasiliano.com.br/revistas/edicao_62.pdf?PHPSESSID=70eda81b51944891dc671955bad21477>.
Acesso em: 18 set. 2012.

BRITO PAIXÃO, Eliana do Socorro de; TOSTES, José Alberto. Laranjal do Jari (estado do Amapá): Conflitos na gestão urbana de uma pequena cidade amazônica e as perspectivas a partir do Plano Diretor Participativo. La planificación territorial y el urbanismo desde el diálogo y la participación. Actas del XI Coloquio Internacional de Geocrítica, Universidad de Buenos Aires, 2-7 de mayo de 2010. Disponível em: http://www.filo.uba.ar/contenidos/investigacion/ institutos/geo/geocritica2010/253.htm. Acesso em: 10 ago 2013.

CASTRO, A. L. C. Glossário de defesa civil: estudo de riscos e medicina de desastres. Brasília: MPO/ Departamento de Defesa Civil, 2008.

COBURN, A.W; SPENCE, R.J.S; POMONIS, A. Vulnerability and Risk Assessment. UNDP Disaster Management Training Program, 1991.

CUTTER, S. L.; BORUFF, B. J. ; SHIRLEY, W. L. Social vulnerability to environmental hazards. Social Science Quarterly, 2003, v. 84 (1): p. 242-261.

GRANZIERA, M. L. M. Direito de Águas. Ed. Atlas, 2001

IBGE. Censo demográfico 2010. Instituto Brasileiro de Geografia e Estatística. 2010. Diponível em: http:// censo2010.ibge.gov.br. Acesso em: 20 ago. 2012.

KUNDZEWICZ, Z. W. Non-Structural Flood Protection and Sustainability. In: Water International. vol.27, n. 1, p. 3-13, Mar, 2002

LUCAS, E.W.M., BARRETO, N. J. C., CUNHA, A. C. Variabilidade hidrológica da Bacia do Rio Jari (AP): Estudo de caso do ano 2000. In: CUNHA., A.C.; SOUZA, E. B.; CUNHA, H. A. C. (orgs.). Tempo, Clima e Recursos Hídricos: resultados do Projeto REMETAP no Amapá: Macapá:IEPA, 2010.

MAKSIMOVIC, C.; TODOROVIC, Z. Non-Structural vs. Structural Alternatives in Sustainable Urban Runoff Management. In: International Workshop on NonStructural Flood Control in Urban Areas. University of São Paulo, 1998.

MARANDOLA JR., E; HOGAN, D. J. Natural hazards: o estudo geográfico dos riscos e perigos. Ambiente \& Sociedade, 2004 p. 95-107.

MARQUES, A. D.; CUNHA. A.C. Valoração de danos sócio-ecônomicos causados por inundação no Município de Laranjal do Jari-AP no ano de 2000. XV Congresso Brasileiro de Meteorologia. Anais em CD ROM, São 
Paulo, SP. 2008.

OLIVEIRA, A.M., CUNHA, A. C. Impactos socioeconômicos associados às enchentes de 2000 e 2006 no município de Laranjal do Jari (AP).In: CUNHA., A.C.; SOUZA, E. B.; CUNHA, H. A. C. (orgs.). Tempo, Clima e Recursos Hídricos: resultados do Projeto REMETAP no Amapá: Macapá:IEPA, 2010.

QUARESMA, Paulo de Souza. Análise da situação dos desabrigados pelas enchentes no município de Laranjal do Jari, estado do Amapá: Uma proposta definitiva (Monografia de especialização). Faculdade Metropolitana de Fortaleza, 2008.

SALGADO, M., G. Reflexões em torno do conceito do risco natural e da dimensão do risco. Faculdade de letras/ Universidade do Porto. 2005

SANTOS, Milton. Técnica, Espaço e Tempo: Globalização e o Meio Técnico-cientifico Informacional. São Paulo. Hucitec, 1998.

SANTOS, Rúbia dos. O Colapso da gestão de crise nas cidades brasileiras. Coimbra: OSIRIS, 2011. Disponível em: http://www.ces.uc.pt/aigaion/attachments/O\%20colapso RubiaSantos.pdf-db48caa580811194f7d79c252426b60d. pdf. Acesso em: 26 nov. 2013.

TOBIN, G. A; MONTZ, B. E., Natural hazards: explanation and integration. The Guilford Press, New York, London. 1997.

TUCCI, C E. M.; VILLANUEVA, A. O. N. Flood control measures in União da Vitoria and Porto Uniao: structural vs. Non-structural measures. In: Urban Water, 2001. p. 177-182. Disponível em: www.elsevier.com/locate/ urbwat. Acesso em: 24 ago.2011.

TUCCI, C. E. M. Aspectos Institucionais do Controle das Inundações Urbanas. In:

TUCCI, C. E. M.; MARQUES, D. M. L. M. (orgs.). Avaliação e Controle da Drenagem Urbana. Porto Alegre: ABRH, 2001 v.2, p. 405-419

UNDP (2004). United Nations Development Programme /Bureau of Crisis Prevention and Recovery. A Global Report Reducing Disaster Risk: A Challenge for Development. UNDP Bureau for Crisis Prevention and Recovery, New York. 2004.

VEYRET, Y. Os riscos: o homem como agressor e vítima do meio ambiente. Contexto, São Paulo. 2007.

ZANIRATO, S.H., RAMIRES, J.Z.S., AMICCI, A.G. N, RIBEIRO, Z.M., RIBEIRO, W.C. Sentidos do Risco:
Interpretações teóricas. Revista Bibliográfica de Geografía e Ciencias Sociales, 2008, vol. 13, no. 785.

ZIMMERMAN, R. The managemet of risk. In. COVELLO, v. t.; MENKES, J.; MUNPOWE, J. Risk evaluation and management. New York: Plenum Press, 1986. P.435-460. 